\title{
A Man With Invasive Community Acquired-MRSA INFECTION IN THE ICU SETTING
}

\author{
Jason Korenblit, $M D, M B A$
}

\section{Case Presentation}

A 66 year-old male presented to the emergency department (ED) with complaints of two days of shortness of breath and pruritis. The patient claimed that he had not had regular medical care for more than 15 years and admitted to no past medical history or chronic medications. One week prior, he reported an itchy rash on both his forearms. He sought treatment at a local free clinic and received a low-potency steroid cream which he used without relief. While at a family event, he became increasingly dyspneic and was brought to the ED by his family. He reported no surgical history and no drug allergies or food intolerances. The patient reported moderate alcohol use with one to two beers per day and denied any tobacco use, substance abuse, or intravenous drug use. He could report no pertinent family history.

On physical examination, the patient appeared mildly dyspneic and uncomfortable, but in no acute distress. He was afebrile, tachycardic with a pulse of 112 beats/minute, tachypnic with respirations of 32 breaths/minute, and normotensive. His lung exam revealed coarse breath sounds bilaterally with decreased respirations at the bases, but no wheeze, rhonchi, or cough. The cardiac exam was within normal limits except for the noted tachycardia, with no murmurs, rubs, or gallops. The abdominal exam revealed a freely reducible left inguinal hernia. Examination of the patient's skin revealed an approximately $15-\mathrm{cm}$ erythematous, non-blanching lesion on his posterior neck with some fluctuance as well as smaller, pustule lesions on his lower back, chest, and arms. The lesions were painless and fluctuant, with no exudates. He was drowsy but oriented, and had no focal neurologic deficits.

The patient's white blood cell count (WBC) was high normal at $10.2 \mathrm{~B} / \mathrm{L}$, hemoglobin was low at $6.3 \mathrm{~g} / \mathrm{dL}$, hematocrit was $19.8 \%$, and platelet count was elevated $534 \mathrm{~B} / \mathrm{L}$. The manual differential of the patient's blood count revealed $61 \%$ neutrophils and $36 \%$ bands. The patient's metabolic panel revealed an elevated potassium of $6.5 \mathrm{mmol} / \mathrm{L}$, very low bicarbonate of $6 \mathrm{mmol} / \mathrm{L}$, a greatly elevated BUN at $198 \mathrm{mg} / \mathrm{dL}$ and a greatly elevated creatinine at $17.8 \mathrm{mg} / \mathrm{dL}$. The patient's lactate was greatly elevated at $26.1 \mathrm{mg} / \mathrm{dL}$. The patient's ESR was $120 \mathrm{~mm} / \mathrm{hr}$. Prothrombin time was prolonged at $19.6 \mathrm{sec}$ which corresponds to an INR of 1.57. Blood cortisol was $117.6 \mu \mathrm{g} / \mathrm{dL}$. An initial venous blood gas revealed a $\mathrm{pH}$ of 7.23 , a pCO2 of 14 , a $\mathrm{pO} 2$ of 131, and a calculated bicarbonate of 7 . Urinalysis revealed a WBC count of 187 per high-powered field, a RBC count of 9 cells per high-powered field, 3+ leukocyte esterase, and many WBC clumps. All other laboratory values including the hepatic function panel were within standard limits. An initial portable chest radiograph revealed questionable bibasilar infiltrates, but was limited by poor inspiratory effort.

The patient was admitted to the medical-respiratory ICU with acute renal failure and a suspected diagnosis of sepsis and

community acquired pneumonia. His PORT score on admission was calculated at 176 , which corresponds to the highest risk class of $\mathrm{V}$ and a greater than $2.8 \%$ in-hospital mortality. The patient was initially treated with aggressive fluid resuscitation, supplemental oxygenation, and the initiation of antibiotics. He received one gram of vancomycin and a renal-adjusted dose of pipericillin-tazobactam and gentamicin due to the severity of his condition. The patient also received kayexalate and several amps of sodium bicarbonate to offset his acidemia and hyperkalemia. Surgery was consulted for possible exploration and debridement of the lesion on the patient's neck.

The morning following the patient's admission to the ICU, initial blood and urine cultures were reported as positive for grampositive cocci in clusters. A non-contrast chest CT (Figure 1) was obtained which demonstrated multi-lobar pneumonia with areas of cavitations as well as a large right pleural effusion and atelectasis. The patient's oxygenation remained stable overnight, but his mental status began to deteriorate. The decision was made at this time to switch the patient's antibiotics to linezolid given the severity of his cavitary pneumonia and the strong suspicion of Staphylococcus aureus infection.

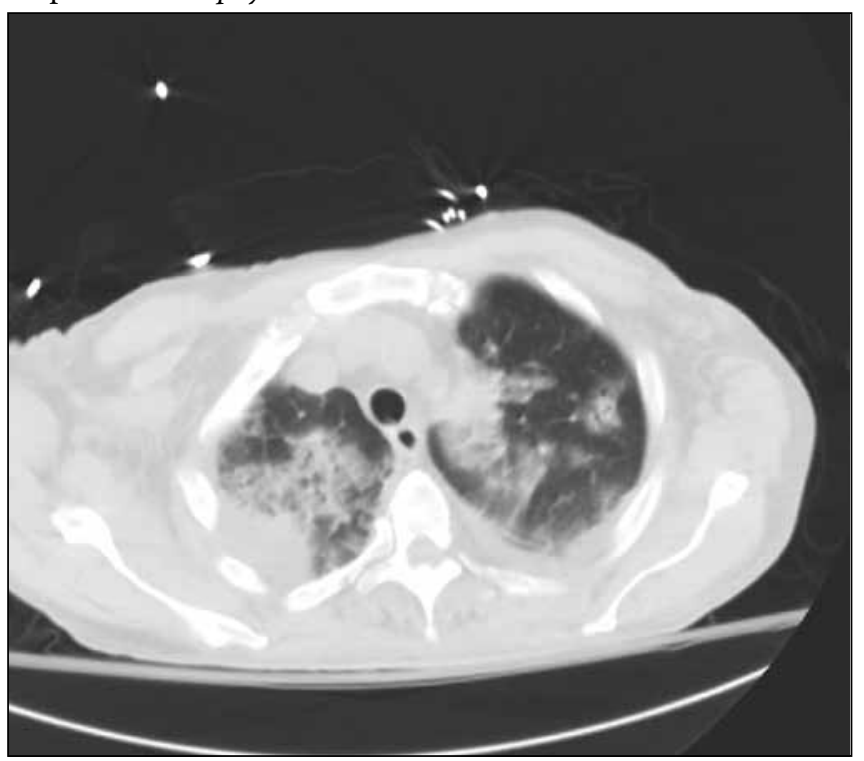

Figure 1. Non-contrast chest CT demonstrating multi-focal cavitary pneumonia and extensive left pleural effusion

Over the next 18 hours the patient's condition deteriorated despite aggressive intervention. Pharmacologic blood pressure support was initiated to combat this patient's sepsis. His renal failure improved but his creatinine was still elevated at 12.6, and his BUN was 165 . Bedside debridement of his neck lesion yielded copious amounts of necrotic material and brown, purulent liquid. Urine culture results returned with confirmation of methicillin- 
resistant Staphylococcus aureus (MRSA), and while sensitivity results were not available yet, it was presumed to be community acquired. Infectious disease consultants advised to switch the patient back to vancomycin given the rapid development of sepsis and hypotension. Pipercillin-tazobactam was continued, and IV azithromycin was added for atypical pneumonia coverage. In addition, oral rifampin was added for synergistic treatment. Clindamycin was debated for its anti-toxigenic properties in the treatment of community acquired MRSA (CA-MRSA), but it was felt that the patient's antibiotic regimen was adequate at the time.

All blood cultures, urine cultures, sputum cultures, and fluid cultures from the surgical debridement grew CA-MRSA within twelve hours of collection despite aggressive, optimal antibiotic treatment. A trans-thoracic echocardiogram revealed a vegetation on the patient's mitral valve with severe damage to the valve and moderate mitral regurgitation. The patient was placed on a bicarbonate drip due to worsening acidemia, and bi-level positive airway pressure was initiated as a non-invasive means of ventilator support.

A meeting with the patient's family was held where it was determined that further aggressive measures would be futile given the patient's continued severe acidemia, renal failure, mental status deterioration, and need for surgical mitral valve replacement. Based on the patient's prior stated wishes and those of his family, no further escalation of care was pursued. The patient expired less than 72 hours from his initial presentation of the ED.

\section{Background and Genetics}

MRSA was first described in Europe in the early 1960's, several years after the introduction of the semi-synthetic beta-lactamase resistant penicillin, methicillin. ${ }^{1}$ It is defined as a Staphylococcus aureus isolate with a minimal inhibitory concentration for oxacillin of greater than 4 micrograms per ml. Approximately $50 \%$ of all $S$. aureus isolates in major teaching institutions are MRSA, resulting in extensive morbidity and mortality from their virulence and multi-drug resistance. ${ }^{2}$ A surveillance report of over 24,000 cases of nosocomial S. aureus bacteremia in the United States revealed that isolates with methicillinresistance increased from $22 \%$ to $57 \%$ between 1995 and $2001 .{ }^{3}$ Worldwide, HA-MRSA prevalence varies considerably, from $<1$ percent in Scandinavia to up to $40 \%$ in Japan, Israel, and Western Europe. ${ }^{4}$

By definition, all strains of MRSA are resistant to beta-lactam antibiotics, including all cephalosporins and extended-spectrum penicillins. The key to this resistance lies in the mec gene, which codes for a novel penicillin-binding protein (PBP 2a) that enables it to resist the binding of all beta-lactam antibiotics. ${ }^{5}$ PBP2a allows the bacterium to assemble its cell wall even in the presence of antibiotics, and the mecA gene complex is freely transmissible in a mobile gene element known as the Staphylococcal cassette chromosome (SCC-mec). In addition to this powerful resistance mechanism, many MRSA isolates produce beta-lactamases as well as various effluent pumps that protect the cell from multiple antibiotics. ${ }^{6}$ Five sub-types of the SCCmec complex have been described; sub-types one, two, and three convey additional multi-drug resistance to quinolones, macrolides, and sulfa-based antibiotics and represent isolates of hospital-acquired MRSA (HA-MRSA). Sub-types four and five represent common $C A-M R S A$ strains without multi-drug resistance, but with added virulence factors that make these strains especially dangerous. ${ }^{7}$

Panton-Valentine leukocidin (PVL) is a unique compound found in CA-MRSA that may be responsible for the added virulence seen in these strains. It is a hemolysin that was first described by Panton and Valentine in 1932 that gives $C A$ $M R S A$ the ability to cause necrotic skin lesions and predisposes for cavitary and hemorrhagic pneumonias, as seen with the index patient described above. ${ }^{7}$ PVL causes transcription-level changes in cells independent of other $S$. aureus toxins and cell products as purified extracts of PVL have been shown to cause pneumonitis in murine models even without the presence of infection. The ability of CA-MRSA to so readily infect immunecompetent hosts may also be attributed to PVL. ${ }^{8}$ One recent study by Moran et al showed that $97 \%$ of CA-MRSA isolates identified at 11 university-affiliated emergency departments were found to produce PVL. ${ }^{9}$

\section{Presentation and Epidemiology}

Presentation of $C A-M R S A$ is usually as a skin and soft tissue infection, but it can cause invasive disease at any site including hematogenous osteomyelitis, septic arthritis, cavitary pneumonia, myositis and pyomyositis, and fasciitis; hematogenous osteomyelitis is the most common invasive infection. ${ }^{8}$ Fridkin et al found that $87 \%$ of cases of CA-MRSA at three community hospitals presented as skin and soft tissue infections or traumatic wound infections, $4 \%$ presented as urinary tract infections, $4 \%$ as sinusitis, $3 \%$ as bacteremia, and only $2 \%$ as community acquired pneumonia. ${ }^{2}$

In a 2003 study by Naimi et al, 75\% of cases of MRSA skin and soft tissue infection were due to CA-MRSA isolates, as opposed to nosocomial HA-MRSA which most commonly presented with bacteremia or catheter infections. ${ }^{8}$ A similar study in 2006 in Los Angeles indicated that a median of $59 \%$ of skin and soft tissue infections presenting to the emergency department were due to $M R S A$, over $95 \%$ of those were considered community acquired strains based on SCCmec genotyping, and 74\% of MRSA isolates were strain U300, a common, PVL-producing strain. ${ }^{9}$

Risk factors for acquiring a $C A-M R S A$ include recent skin trauma, high body mass index, practice of cosmetic body shaving, male gender, participation in contact sports, prolonged hospitalization, incarceration, military service, diabetes, intravenous drug use, and history of a prior skin infection. No study has consistently shown risk factors that define MRSA infections 
versus other skin and soft tissue infections; therefore empiric coverage should always be considered. ${ }^{10}$

\section{Treatment}

Treatment of CA-MRSA is oftentimes more straightforward since these particular strains do not exhibit multi-drug resistance that nosocomial MRSA innately display. Retrospective studies of CA-MRSA soft tissue infections have shown $95 \%$ were susceptible to clindamycin, $6 \%$ to erythromycin, $60 \%$ to fluoroquinolones, $100 \%$ to rifampin and trimethoprim-sulfamethoxazole (TMPSMX), and $92 \%$ to tetracycline. ${ }^{9}$ Although proper antibiotic choice is an important part of treatment protocol to prevent further progression to invasive disease, the most important treatment of focal skin involvement is adequate drainage of any abscess or infectious collection. A 2007 prospective trial found that lack of incision and drainage was associated with significant non-response 30 days after initial treatment. Current CDC recommendations state that oral clindamycin or oral TMPSMX should be first-line for empiric coverage of presumed $C A$ $M R S A$ for all skin and soft-tissue infections identified. ${ }^{12}$

Intravenous antibiotic therapy for invasion $M R S A$, whether nosocomial or community acquired, centers on vancomycin and achieving appropriate therapeutic levels in target tissue. Vancomycin inhibits cell wall synthesis by preventing $\mathrm{N}$ acetylmuramic acid (NAM)- and N-acetylglucosamine (NAG)-peptide subunits from being incorporated into the peptidoglycan matrix. It remains the antibiotic of choice for treatment of invasive $M R S A$ infections, given its relatively good safety profile and favorable pharmacokinetics that facilitate convenient administration. In addition, vancomycin is the agent for which there is the greatest cumulative clinical experience for the treatment of a variety of invasive clinical syndromes including pneumonia, endocarditis, meningitis, and osteomyelitis. ${ }^{13}$ Reports of MRSA with either intermediate or full resistance to vancomycin or high in vitro resistance have spurred research for newer alternative agents.

Daptomycin is a novel intravenous agent used to combat MRSA and vancomycin-resistant enterococcus (VRE). It is a cyclic lipopeptide bactericidal antibiotic that causes depolarization of the bacterial cell membrane and is used for bacteremia, endocarditis, and soft-tissue infections due to invasive nosocomial and community acquired MRSA. ${ }^{14}$ Heteroresistance to daptomycin may develop during treatment, especially with co-exposure to vancomycin, so susceptibility testing may be necessary during treatment. Daptomycin is also inactivated by surfactant and is thus ineffective in treating pneumonia caused by $M R S A .{ }^{15}$

Linezolid is a bacteriostatic oxazolidinone antibiotic that inhibits initiation of protein synthesis at the $50 \mathrm{~S}$ ribosome, and does not exhibit cross resistance with other protein synthesis inhibitors. Its mechanism of action may lead to enhanced efficacy against strains producing toxins such as PVL, alpha-hemolysin, and toxic-shock syndrome toxin-1, making it an excellent choice for initial therapy in severe cases of $C A-M R S A .{ }^{16}$ Linezolid has excellent tissue distribution, especially in the lung parenchyma, and may be administered parenterally or orally due to its high bioavailability.

Other potential treatment options include tigecycline, a novel tetracycline derivative that is active against most gram-positive infections. Tigecycline mono-therapy was deemed as effective as vancomycin plus aztreonam for complicated skin and soft tissue infections in a prospective randomized trial. ${ }^{17}$ Quinupristindalfopristin, a streptogramin antibiotic whose use is limited by side effect profile and high cost, has shown efficacy when used to treat patients who have failed vancomycin. One study by Drew et al showed $71 \%$ response in invasive infections in patients who could not tolerate or failed vancomycin therapy. ${ }^{18}$

Several investigational agents are undergoing trials. Dalbavancin is a semi-synthetic lipoglycopeptide that has been demonstrated to be at least as effective as vancomycin and linezolid, but has a half-life of six to twelve days and can be dosed once per week. ${ }^{19}$ Ceftobiprole is an extended-spectrum cephalosporin similar to cefepime that can circumvent PBP2a that confers betalactam resistance to $M R S A$. A randomized trial of 828 patients demonstrated efficacy similar to vancomycin plus ceftazadime. ${ }^{20}$ Preparations of staphylococcal antibodies have been investigated for the treatment of $S$. aureus bacteremia. Tefibazumab is a monoclonal antibody that targets clumping factor A, a protein on the surface of $S$. aureus that binds to human fibrinogen. Its current efficacy is debatable. ${ }^{21}$ Altastaph is an agent consisting of a pooled group of antibodies to $S$. aureus capsular polysaccharide types five and eight and may serve as an adjuvant therapy. ${ }^{22}$

\section{Prevention}

Prevention of $C A-M R S A$ infection remains a community health challenge as few therapies have proven effective. No trial data supports the use of empiric nasal mupiracin or topical chlorhexadine rinse to prophylax against infection. ${ }^{12}$ Hand washing and avoidance of contact with infected individuals is key. Empiric treatment of close contacts of an infected individual is controversial, and no compelling data exists at this time. Pets have also been offered as a possible source of bacterial reservoir, and while transmission from an infected pet is possible, eradication therapy in household pets is not currently recommended. ${ }^{12}$

\section{References}

1. Benner, EJ, Kayser, FH. Growing clinical significance of methcillin-resistant Staphylococcus aureus. Lancet 1968; 2:741

2. Fridkin et al. Methicillin-Resistant Staphylococcus aureus Disease in Three Communities NEJM 2005;352:1436-44

3. Wisplinghoff $\mathrm{H}$, et al. Nosocomial bloodstream infections in US hospitals: analysis of 24,179 cases from a prospective nationwide surveillance study. Clin Infect Dis 2004 Aug 1;39(3):309-17. Epub 2004 Jul 15

4. Sader HS, et al. Antimicrobial susceptibility of gram-positive bacteria isolated 
from European medical centres: results of the Daptomycin Surveillance Programme (2002-2004). Clin Microbiol Infect. 2006 Sep;12(9):844-52.

5. Enright, et al. The evolutionary history of methicillin-resistant Staphylococcus aureus (MRSA) Proc Natl Acad Sci 2002; 99:7687

6. Hanaki $\mathrm{H}$, Labischinski $\mathrm{H}$, Inaba $\mathrm{Y}$, et al: Increase in glutamine-non-amidated muropeptides in the peptidoglycan of vancomycin-resistant Staphylococcus aureus strain Mu50. J Antimicrob Chemother 1998; 42:315-320.

7. Mandell, Bennett, \& Dolin: Principles and Practice of Infectious Diseases, 6th ed Chapter 192.

8. Naimi T.S., LeDell K.H., Como-Sabetti K., et al: Comparison of community- and health care-associated methicillin-resistant Staphylococcus aureus infection. JAM A 290. 2976-2984.2003

9. Moran GJ et al. Methicillin-resistant S. aureus infections among patients in the emergency department. N Engl J Med. 2006 Aug 17;355(7):666-74.

10. Calfee, DP, Durbin, LJ, Germanson, TP, et al. Spread of methicillin-resistant Staphylococcus aureus (MRSA) among household contacts of individuals with nosocomially acquired MRSA. Infect Control Hosp Epidemiol 2003; 24:422

11. Miller LG et al. A prospective investigation of outcomes after hospital discharge for endemic, community-acquired methicillin-resistant and -susceptible Staphylococcus aureus skin infection. Clin Infect Dis. 2007 Feb 15;44(4):483-92. Epub 2007 Jan 12

12. Daum R. Methicillin resistant Staphylococcus aureus in the community: infection control strategies. Program and abstracts of the 44th Interscience Conference on Antimicrobial Agents and Chemotherapy; October 30-November 2, 2004; Washington, DC. Session 153; Abstract 1464

13. Mohr JF; Murray BE. Point: Vancomycin is not obsolete for the treatment of infection caused by methicillin-resistant Staphylococcus aureus. Clin Infect Dis. 2007 Jun 15;44(12):1536-42. Epub 2007 May 4.

14. Arbeit RD, et al. The safety and efficacy of daptomycin for the treatment of complicated skin and skin-structure infections. Clin Infect Dis 2004 Jun 15;38(12):1673-81. Epub 2004 May 20.

15. Sakoulas G, et al. Induction of daptomycin heterogeneous susceptibility in Staphylococcus aureus by exposure to vancomycin. Antimicrob Agents Chemother. 2006 Apr;50(4):1581-5.

16. Weigelt $\mathrm{J}$, et al. Linezolid versus vancomycin in treatment of complicated skin and soft tissue infections. Antimicrob Agents Chemother 2005 Jun;49(6):2260-6.

17. Ellis-Grosse EJ, et al. The efficacy and safety of tigecycline in the treatment of skin and skin-structure infections: results of 2 double-blind phase 3 comparison studies with vancomycin-aztreonam. Clin Infect Dis 2005 Sep 1;41 Suppl 5: S341-53.

18. Drew RH, et al. Treatment of methicillin-resistant staphylococcus aureus infections with quinupristin-dalfopristin in patients intolerant of or failing prior therapy. For the Synercid Emergency-Use Study Group. J Antimicrob Chemother 2000 Nov;46(5):775-84

19. Jauregui LE, et al. Randomized, double-blind comparison of once-weekly dalbavancin versus twice-daily linezolid therapy for the treatment of complicated skin and skin structure infections. Clin Infect Dis 2005 Nov 15;41(10):1407-15. Epub 2005 Oct 6 .

20. Noel GJ, et al. A randomized, double-blind trial comparing ceftobiprole medocaril with vancomycin plus ceftazidime for the treatment of patients with complicated skin and skin-structure infections. Clin Infect Dis. $2008 \mathrm{Mar}$ $1 ; 46(5): 647-55$

21. Weems JJ Jr, et al. Phase II, randomized, double-blind, multicenter study comparing the safety and pharmacokinetics of tefibazumab to placebo for treatment of Staphylococcus aureus bacteremia. Antimicrob Agents Chemother. 2006 Aug;50(8):2751-5.

22. Rupp ME, et al. Phase II, randomized, multicenter, double-blind, placebocontrolled trial of a polyclonal anti-Staphylococcus aureus capsular polysaccharide immune globulin in treatment of Staphylococcus aureus bacteremia. Antimicrob Agents Chemother. 2007 Dec;51(12):4249-54. Epub 2007 Sep 24.

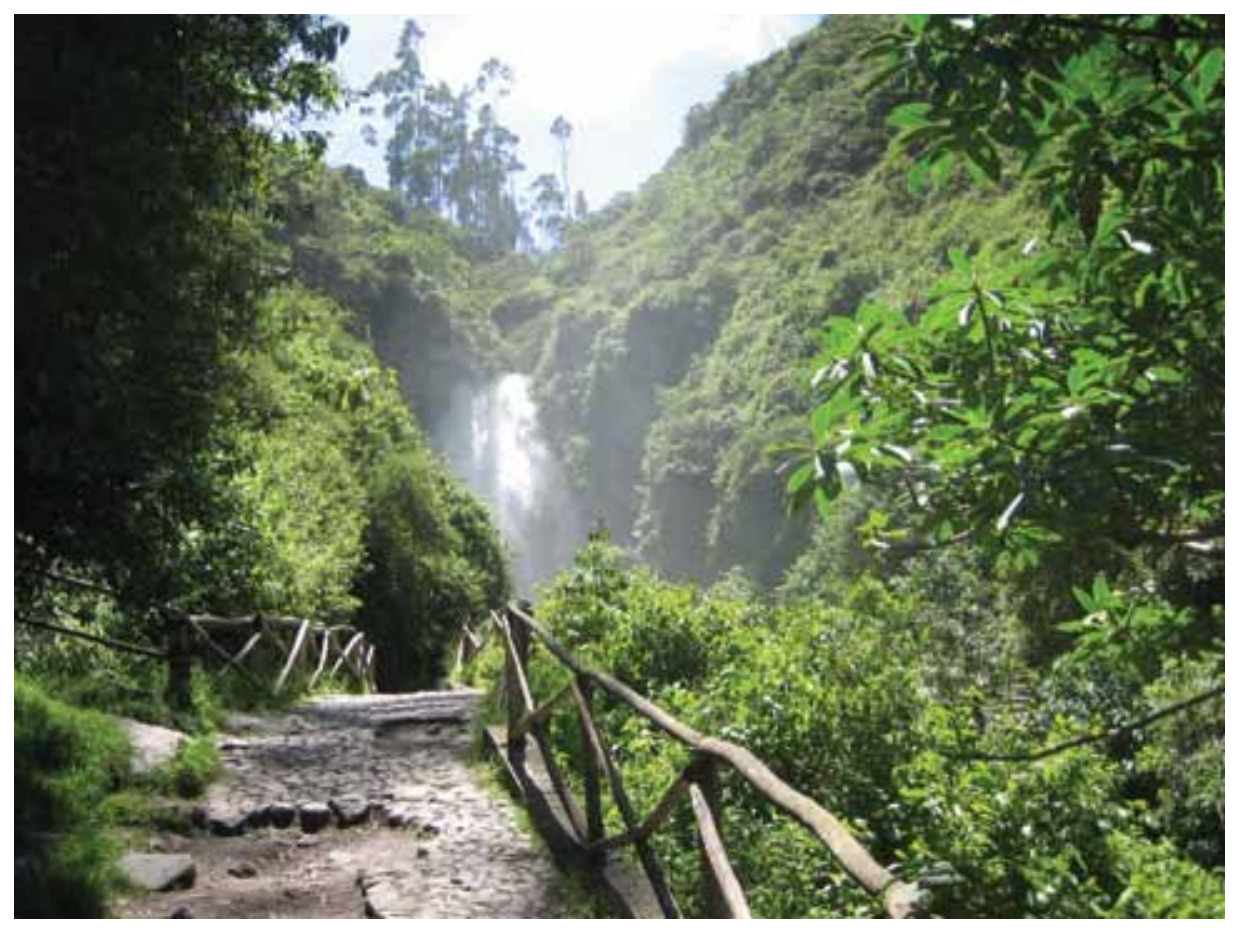

Photograph courtesy of

Sandarsh Kancherla, MD 